\title{
AVLSI Compatible Conducting Polymer Composite Based "Electronic Nose" Chip
}

\author{
Nathan S. Lewis \\ Professor of Chemistry \\ Division of Chemistry and Chemical Engineering, 127-72 \\ Noyes Laboratory \\ Pasadena, CA 91125
}

\begin{abstract}
The focus of this work would be to exploit the vapor detection technology developed recently at Caltech that forms the basis for a low power, simple "electronic nose". In this work we have integrated the sensors, signal preprocessing, signal processing, and data analysis functions into a single, low power, low cost, "nose chip". Such a device could be implantable covertly or overtly onto suspect sites, deployable through remote delivery methods, worn by soldiers for $\mathrm{CW}$ alerts and in principle for IFF or military/nonmilitary identification purposes, and for other areas of national security where low power, lightweight, small, chemical sensing is of importance.
\end{abstract}

\section{INTRODUCTION}

The basis for our approach involves an effort at Caltech that has recently led to arrays of simple, readily fabricated, chemically sensitive conducting polymer films (1-3). An array of sensors that individually respond to vapors can produce a distinguishable response pattern for each separate type of analyte or mixture. Pattern recognition algorithms and/or neural network hardware are used on the output signals arising from the electronic nose to classify, identify, and where necessary quantify, the vapor or odors of concern (4-5). This response is much like the way the mammalian olfactory sense produces diagnostic patterns and then transmits them to the brain for processing and analysis.

The underlying principle of the Caltech electronic nose is extraordinarily simple. When a polymer film is exposed to a gaseous vapor, some of the vapor partitions into the film and causes the film to swell (Fig la). This swelling is probed electrically because the sensor films each consist of a composite that contains regions of a conductor that have been dispersed into the swellable organic insulator. The vapor-induced film swelling produces an increase in the electrical resistance of the film because the swelling decreases the number of connected pathways of the conducting component of the composite material. Any individual sensor film responds to a variety of vapors, because numerous chemicals will partition into the polymer and cause it to swell to varying degrees (Fig 1b) (6).

However, an array of sensors, containing different polymers, yields a distinct fingerprint for each odor because the swelling properties over the entire array are different for different vapors.

The pattern of resistance changes on the array is diagnostic of the vapor, while the amplitude of the patterns indicates the concentration of the vapor. An example of the different patterns produced by various different vapors on the electronic nose is provided in Fig. 2. The swelling/deswelling process is highly reversible in thin polymer films, so that typically thousands of cycles can be performed without significant loss of responsivity of the detectors. The detectors have been shown to respond to analyte concentrations in the low ppb range under certain conditions, with more specific chemistries allowing detection in targeted cases, such as biogenic amines for health/disease differentiation, in the ppt range.

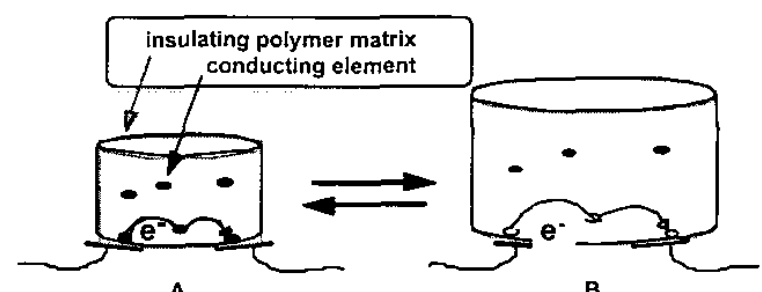

(a)

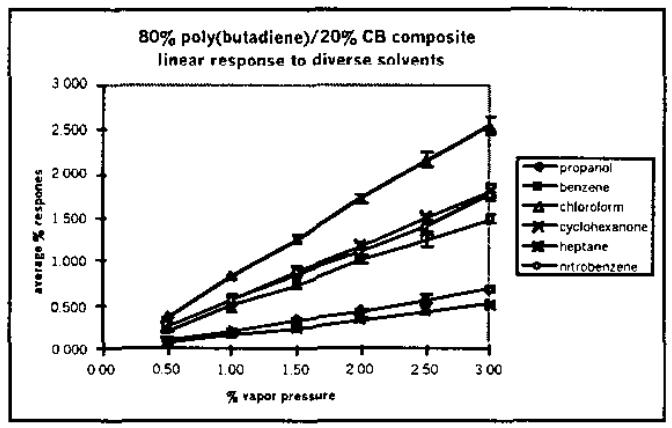

(b)

Fig. la Swelling occurs as an odorant partitions into the polymer. (b) A linear response of an individual sensor signal as a function of concentration is observed for a variety of analytes. 


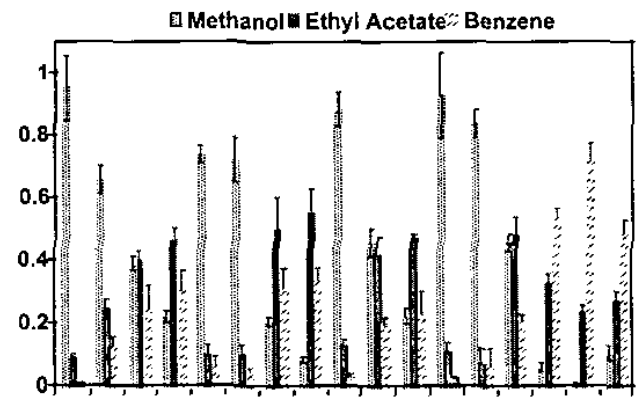

Sensor Number (normalization factor)

(a)

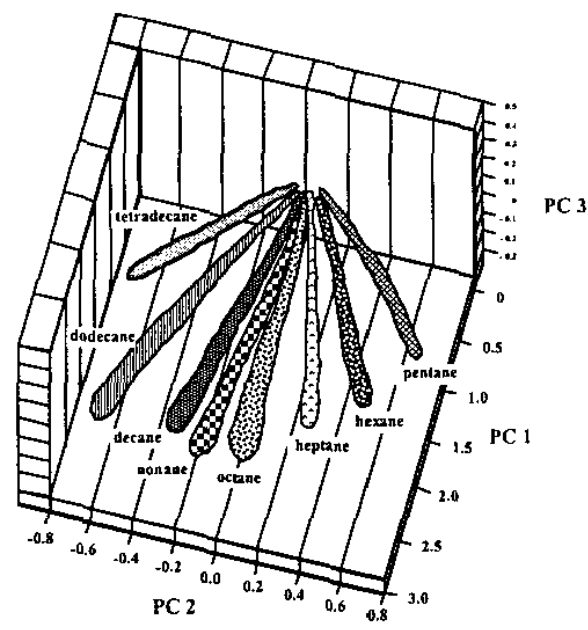

(b)

Fig. 2a. Response patterns for three different solvents on a 17 element sensor array. (b) Data in principal component space from a 20-detector array exposed to $n$-tetradecane, $n$-dodecane, n-decane, n-nonane, n-octane, and $\mathrm{n}$-heptane each at $\mathrm{P} / \mathrm{P}^{\circ}=0.005$ to 0.03 in air (with $\mathrm{P}^{\circ}$ being the vapor pressure of the analyte at $300 \mathrm{~K}$ ), showing that the pattern type identifies the vapor and the magnitude of the pattem signals is linearly proportional to the analyte concentration.

We have fabricated "nose-chips", which consist of arrays of pixels in which each pixel contains a different composition of polymeric sensor and the signals are fed to a common pinout using a simple row/column multiplexing addressing scheme. This chip is a component of an integrated system, all on one chip, that contains the entire functionality spanning sample introduction, data acquisition, signal preprocessing, adaptation, filtering, and signal processing functions. Development of the necessary compatible technologies, along with their integration, and determination of the performance of the resulting "nose chip" system for targeted applications of interest to national security applications, is the focus of some of the future work to be discussed in this presentation.

\section{R\&D GOALS/APPROACH}

We have fabricated some preliminary "nose-chips", which consist of arrays of pixels in which each pixel contains a different composition of polymeric sensor and the signals are fed to a common pinout using a simple row/column multiplexing addressing scheme (Fig. 3). This chip demonstrates the validity of the approach but is not an integrated system, all on one chip, that contains the entire functionality spanning sample introduction, data acquisition, signal preprocessing, adaptation, filtering, and signal processing functions. Development of the necessary compatible technologies, along with their integration, and determination of the performance of the resulting "nose chip" system for targeted applications of interest to national security applications, will be discussed.

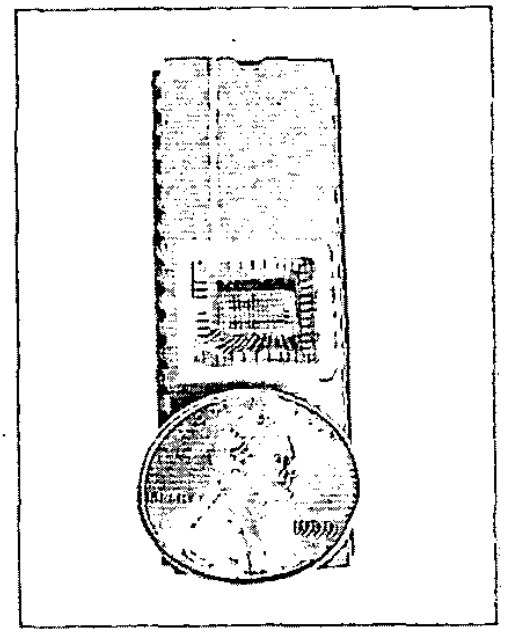

Fig. 3. Photograph of a "nose-chip", with 12 columns and 6 rows, each having a different polymeric sensor combination and each pixel having a switch under its pair of contact lines. A single pinout provides the output of the sensor pixel being interrogated using a row/column addressing scheme.

Figure 4 shows the schematic of the unit sensor cell. The cell consists of a switch transistor and decoding logic. The availability of only two metals layers in the IC process required transistors at each sensor cell to perform decoding. This circuitry (M1-M4) decodes X and Y selection signals generated by shift registers on the periphery of the array. This selection signal controls a switch (M7) that toggles a current $\left(\mathrm{I}_{\mathrm{in}}\right)$ through the resistive sensor. In this design only one sensor is energized at a time to reduce power consumption. To reduce noise and the switch resistance, transistor M7 occupies most of the sensor area. The decoding circuitry also selects a transmission gate 
(M5,M6,M8,M9) which passes the sensor voltage to a column output bus. This signal is amplified and transmitted off-chip for processing. The decoding circuitry is complicated because the sensor occupies one of two available metal layers in the fabrication process we used for this chip, precluding the use of a simplified bus scheme.

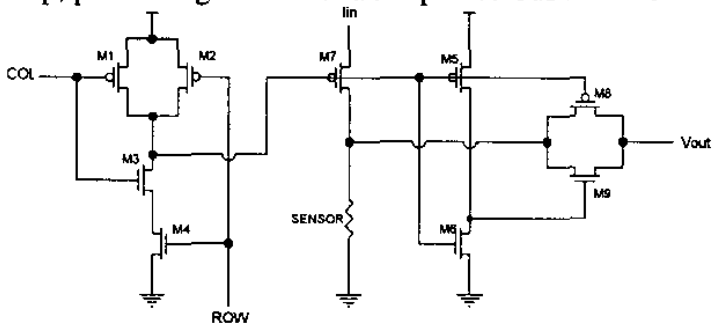

Figure 4. Schematic of three wire sensing cell. Transistors M1-M4 form a NAND gate to select the cell, $M 7$ switches the current source on sensor resistor, and M5,M6,M8,M9 form a transmission gate to select the output on a column output line. The column output is buffered and passed offchip.

A $0.5 \mathrm{~cm} \times 0.25 \mathrm{~cm}$ chip, with 492 sensors arranged as an array of $41 \times 12$ sites, was fabricated in a 2.0 micron process through the MOSIS design service. There are two contacts to the sensor: one with the drive switch $\mathrm{M} 7$, and another connecting the sensor to signal ground. The ground terminal is laid out as a ring around the perimeter of the cell and is common to all sensors. The interior of each cell contains the drive contact for the sensor. The ring structure was motivated by difficulties encountered in early sensor deposition trials - the carbon black particles would aggregate along the perimeter of a deposited sensor, creating a low resistance path. Moreover, the ring structure allows us to experiment with depositing mixtures of sensor materials across the chip[5]. We deposit the sensor polymer between these two contacts, directly on top of the active circuitry. We make the sensors rectangular to increase the contact area for the interior contact, as well as to reduce contact noise and $1 / \mathrm{f}$ noise due to the non-uniform electrical field[6].

We use a standard commercial foundry for the fabrication of the integrated circuits. The top layer of Aluminum is used for the sensor contact. Unfortunately, the native aluminum oxide that forms on the contacts prevents depositing the sensors without a post-processing step. Since dedicated wafer runs are cost prohibitive for small prototyping runs, this step must be performed on the individual chip die returned from the foundry. This precludes the use of a conventional mask based approach, since it is difficult to use a resist mask on an individual die.

To create suitable contacts we use an electroless $\mathrm{Ni} / \mathrm{Au}$ process that requires no masking from Stapleton Technologies (Long Beach, California). This process can be performed easily on individual die with simple equipment and requires only seven procedures: four involving cleaning and surface preparation and three plating steps. The surface preparation involves an acid zincate process to remove the native oxide and activate the aluminum surface. This is followed by the three plating steps. Nickel is plated first, followed by a two stages of gold plating: a monolayer process that plates the Nickel and then a build up stage that finishes the plating. In addition to creating a nonreactive surface for the sensor contacts, the plating also creates wells that help constrain the sensor material during deposition.

\section{CONCLUSION}

We have demonstrated the successful integration of a chemical sensor array with a standard CMOS process. Following a simple post processing operation we are able to deposit sensor material on the surface on an integrated circuit. By depositing different sensor materials we are able to create an array capable of discriminating analytes. We plan to create larger arrays with a large number of different polymers, and arrays with additional active circuitry such as amplification and adaptation. Our ultimate goal is a chip with several thousand integrated sensors, combined with active processing circuitry. Intimate integration of active circuitry and sensor not only enables large arrays, but also is essential in overcoming the historical limitations of polymer-based sensors. We believe that our approach can create small, inexpensive, low power and even wearable chemical sensor arrays that rival the detection and discrimination capabilities of mammalian olfaction.

\section{REFERENCES}

(1) M.C. Lonergan, E.J. Severin, B.J. Doleman, S.A. Beaber, R.H. Grubbs, and N.S Lewis, "Array-Based Vapor Sensing Using Chemically Sensitive, Carbon Black-Polymer Resistors," Chem. Mater, vol. 8, pp. 2298-2312, 1996.

(2) E.J. Severin, B.J. Doleman, and N.S. Lewis, "An Investigation of the Concentration Dependence and Response to Analyte Mixtures of Carbon Black/Insulating Organic Polymer Composite Vapor Detectors," Anal. Chem., vol. 72, pp. 658-668, 2000.

(3) B.J. Doleman, R.D. Sanner, E.J. Severin, R.H. Grubbs, and N.S. Lewis, "Use Of Compatible Polymer Blends To Fabricate Arrays Of Carbon Black-Polymer Composite Vapor Detectors," Anal. Chem., vol. 70, pp. $2560-2564,1998$.

(4) B.J. Doleman, M.C. Lonergan, E.J. Severin, T.P. Vaid, and N.S Lewis, "Quantitative Study of the Resolving Power of Arrays of Carbon Black-Polymer Composites in Various Vapor-Sensing Tasks," Anal. Chem., vol. 70, pp. 4177-4190, 1998.

(5) T.P. Vaid, M.C. Burl, and N.S. Lewis, "Comparison of the Performance of Different Discriminant Algorithms in Analyte Discrimination Tasks Using an Array of Carbon Black-Polymer Composite Vapor Detectors," Anal. Chem., vol 73, pp. 321-331, 2001 .

(6) E.J. Severin and N.S. Lewis, "Relationships among Resonant Frequency Changes on a Coated Quartz Crystal Microbalance, Thickness Changes, and Resistance Responses of Polymer-Carbon Black Composite Chemiresistors," Anal. Chem., vol. 72, pp. 20082015,2000 . 